\title{
The Quantization Relations for the Metric Tensor of Gravitons
}

\author{
Piero Chiarelli \\ National Council of Research of Italy, Pisa, Italy \\ Email: pchiare@ifc.cnr.it
}

How to cite this paper: Chiarelli, P. (2018) The Quantization Relations for the Metric Tensor of Gravitons. Open Access Library Journal, 5: e4872.

https://doi.org/10.4236/oalib.1104872

Received: August 29, 2018

Accepted: September 23, 2018

Published: September 26, 2018

Copyright $\odot 2018$ by author and Open Access Library Inc.

This work is licensed under the Creative Commons Attribution International License (CC BY 4.0).

http://creativecommons.org/licenses/by/4.0/

\section{(c) (i) Open Access}

\begin{abstract}
By using the gravity equation for quantum mechanical systems that takes into account the non-local interaction of the quantum potential the paper derives the quantization of the graviton in the limit of weak gravity. The output of the theory shows that, in the non-Minkowskian quantum approach proposed, where the coupling between the gravitational equation and the field is explicitly defined, a massless boson field can be associated to the graviton. The paper shows that the commutation relations of the metric tensor of the gravitational waves can be analytically derived by the quantization of the associated graviton field.
\end{abstract}

\section{Subject Areas}

Modern Physics

\section{Keywords}

Graviton, Background Quantization, Quantum Gravity, Graviton Quantum Field

\section{Introduction}

The definition of a satisfying quantum gravity [1] requires a clear and not-contradictory connection between the quantum fields theory (QFT) and the general relativity. The problem has come to a partial solution in the semiclassical approximation, where the energy-impulse tensor density (EITD) is substituted by its expectation value, leading to the explanation of the Hawking radiation and $\mathrm{BH}$ evaporation [2]-[7]. But this is far from being the real solution of the problem since this approach does not introduce into the classical gravity the non-local interaction of the quantum mechanics whose most important expressions are the quantum uncertainty and the coherent evolution of the quantum 
superposition of states. In one word, the Einstein equation is not able to support the quantum evolution of state and to describe a self-consistent quantum gravitational physics.

The difficulties become clear in the so called cosmological constant (CC) problem that Einstein added to its equation to give stability to the solution of universe evolution. The introduction, by hand, of the cosmological constant was then refused by Einstein himself as the "biggest mistake of my life" [8]. Actually, the CC is basically assumed by semiempirical arguments [1] as a pressure term in the EITD of the Einstein equation. Moreover, for classical bodies the EITD is also defined in a semiempirical way [9] without any analytical connection with the quantum fields that originate the matter. As discussed by Thiemann [10], this connection cannot be build up by simply replacing the EITD with its Minkowski expectation value. If we do so, we end with a non-Minkowskian metric tensor that has to feed back into the vacuum expectation value and so on with the iteration that does not converge in general.

Due to this undefined connection between the gravity equation (GE) and the particle fields, the integration between QFT and the GE is under intense theoretical investigation. As analytically discussed by Rugh and Zinkernagel [1], there is a defined convincement in the scientific community about the solution of the problem that can be achieved by the inspection of three items.

1) Definition of an adequate new gravity equation (GE) that takes into account the quantum non-local interaction;

2) Definition of the QFT at high energies;

3) Definition of the link between the GE and the QFT.

At glance with the point one, by using the principle of minimum action, the author has derived a GE from the hydrodynamic representation of quantum mechanics [11] that contains the non-local interactions of the quantum mechanics and gives an analytical connection with the quantum fields that are present in the space-time [12].

The modified quantum mechanical gravity equation (QMGE)) [11], takes into account the non-local interaction through the quantum potential, a kinetic form of energy, that in addition to the classical energies defines the physical evolution and, hence, contributes to the space-time curvature. The quantum potential contribution gives rise to a cosmological energy-impulse tensor density (CEITD) that in the classical limit reduces to the constant (i.e., the cosmological one). The CEITD has the remarkable feature of possibly leading to the reduction of the discrepancy between the enormous cosmological constant value deriving by the zero-point vacuum energy density of the QFT (uniformly distributed into the space, following the prescription of the Einstein gravity equation (EGE)) with respect the observed one deriving by the astronomical observations [13] [14]. Moreover, due to the properties of the quantum potential, in the quasi-Minkowskian limit the proposed modified QMGE leads to a renormalizable gravity [12] at glance with the QFT. 
Since the QMGE contains the explicit coupling with the quantum fields, the commutation rules for the metric tensor remains defined as a consequence of the field quantization commutators. In this paper the QMGE equation is used to derive the commutations rules for the quantization of the metric e tensor in the case of massless gravitons.

\section{The Coupling of the QMGE with Quantum Fields}

The QMGE, that includes the quantum potential energy for the definition of the space-time geometry, derived in [11], reads

$$
R_{\mu \nu}-\frac{1}{2} R g_{\mu \nu}-\frac{8 \pi G}{c^{4}}\left(\bar{T}_{\mu \nu}+\Lambda g_{\mu \nu}\right)=0
$$

where

$$
\begin{gathered}
\bar{T}_{\mu v}=T_{\mu v}-\frac{4}{3} g_{\mu \nu} T_{\beta}^{\beta}=-|\psi|^{2} \dot{q}_{\mu} p_{v}, \\
T_{\mu}{ }^{v}=-|\psi|^{2}\left(\dot{q}_{\mu} \frac{\partial L}{\partial \dot{q}_{v}{ }^{(k)}}-L \delta_{\mu}{ }^{\nu}\right)
\end{gathered}
$$

where $L=-p_{\mu} \dot{q}^{\mu}$, where $\dot{q}^{\mu}$ and $p_{\mu}=-\frac{\partial S}{\partial q^{\mu}}$ are given by the solution of the quantum hydrodynamic problem [11] [12] [15] (i.e., $\psi=|\psi| \exp \frac{i}{\hbar} S$ and $L=\frac{\mathrm{d} S}{\mathrm{~d} t}$, see below (8)-(9)) and where the cosmological energy-impulse tensor density(CEITD) $\Lambda g_{\mu v}$ reads

$$
\Lambda g_{\mu v}=\left(T_{\mu v}-\bar{T}_{\mu v}\right)+T_{\text {class } \beta}^{\beta} g_{\mu v}=\left(-\frac{1}{3} T_{\beta}^{\beta}+T_{\text {class } \beta}^{\beta}\right) g_{\mu v}
$$

where

$$
T_{\text {class } \beta}^{\beta}=\lim _{\hbar \rightarrow 0} \bar{T}_{\beta}^{\beta} .
$$

For k-plane waves, Equation (4) reads

$$
\Lambda_{(k)}=( \pm) \frac{m c^{2}}{\gamma}\left|\psi_{ \pm k}\right|^{2}\left(1-\sqrt{1-\frac{V_{q u(k)}}{m c^{2}}}\right)
$$

where

$$
V_{q u}=-\frac{\hbar^{2}}{m} \frac{1}{|\psi| \sqrt{-g}} \partial_{\mu} \sqrt{-g} g^{\mu v} \partial_{\nu}|\psi|
$$

where $\psi$ obeys to the Klein Gordon equation (KGE) that in the hydrodynamic representation leads to the system of equations [11] [12]

$$
\begin{gathered}
g_{\mu \nu} \partial^{\nu} S \partial^{\mu} S-\hbar^{2} \frac{1}{|\psi| \sqrt{-g}} \partial_{\mu} \sqrt{-g}\left(g^{\mu \nu} \partial_{\nu}|\psi|\right)-m^{2} c^{2}=0 \\
\frac{1}{\sqrt{-g}} \frac{\partial}{\partial q^{\mu}} \sqrt{-g}\left(g^{\mu \nu}|\psi|^{2} \frac{\partial S}{\partial q^{v}}\right)=0
\end{gathered}
$$




\section{The Energy-Impulse Tensor Density of Boson Field}

In order to quantize the (scalar uncharged) field $\psi$ obeying to the free KGE in the non-Minkowskian geometry defined by the QMGE (1), we need to express the energy impulse tensor density (EITD) in (1) as a function of such field $\psi$ that by using the expression (3), for the Fourier superposition of plane waves, see [12]

$$
T_{\mu v}=\frac{|\psi|^{2}}{2} \frac{\sum_{k=0} a_{k}\left|\psi_{k}\right| \exp \left[\frac{i S_{k}}{\hbar}\right] \mathrm{T}_{(k) \mu v}}{\sum_{k=0} a_{k}\left|\psi_{k}\right| \exp \left[\frac{i S_{k}}{\hbar}\right]}+\frac{|\psi|^{2}}{2} \frac{\sum_{k=0} a_{k}^{*}\left|\psi_{k}\right| \exp \left[\frac{-i S_{k}}{\hbar}\right] \mathrm{T}_{(k) \mu v}}{\sum_{k=0} a_{k}^{*}\left|\psi_{k}\right| \exp \left[\frac{-i S_{k}}{\hbar}\right]}
$$

where

$$
\mathrm{T}_{(k) \mu \nu}=-c^{2}\left(\frac{\frac{\hbar}{2 i} \partial \ln \left[\frac{\psi_{k}}{\psi_{k}^{*}}\right]}{\partial t}\right)^{-1}\left(\left(\frac{\hbar}{2}\right)^{2} \frac{\partial \ln \left[\frac{\psi_{k}}{\psi_{k}^{*}}\right]}{\partial q^{\mu}} \frac{\partial \ln \left[\frac{\psi_{k}}{\psi_{k}^{*}}\right]}{\partial q^{v}}+m^{2} c^{2}\left(1-\frac{V_{q u(k)}}{m c^{2}}\right) g_{\mu v}\right)
$$

In the case of quantized fields $a_{n}$ and $a_{n}{ }_{n}$ are quantum operators [12].

\section{The Graviton in the Hydrodynamic GE}

In the hydrodynamic quantum gravity approach, the fields generate quantized EITDs that define stationary geometries of the space-time. Moreover, when such fields are quantized and the EITD, on the right side of the QMGE, becomes a quantum operator [12], also the curvature tensor on the left side acquires the properties of a quantum operator.

This also happens for massless particles such as the photon.

As we can check at the end, the graviton can be assumed massless in the limit of quasi-Minkowskian space-time curvature wave. The massless condition can be implemented by requiring the null trace of the Ricci tensor (i.e., scalar curvature) in the QMGE that reads

$$
R=-\frac{8 \pi G}{c^{4}} T_{(n)}=0
$$

If for the photon the field is given by the EM vector potential, for the graviton, where only the gravitational interaction is considered, the boson field can be defined as that one that produces the space-time wave curvature. This assumption is retained by assuming a principle of "specularity" between fields and space-time geometry in general relativity by which any presence of particle (field) generates a space-time curvature and, vice versa, any space-time curvature owns a field (i.e., a particle (even massless)).

In the following we are going to show that the graviton can be described by a boson field $\psi_{\alpha \beta}$ (associated to the space-time gravitational wave $\varepsilon_{\mu \nu}$ ) that obeys to the Klein-Gordon equation (KGE)

$$
\left(\partial^{\mu} \psi_{\alpha \beta}\right)_{; \mu}+\frac{m_{G}{ }^{2} c^{2}}{\hbar^{2}} \psi_{\alpha \beta}=0
$$


coupled to the QMGE

$$
R_{\mu v}-\frac{1}{2} R g_{\mu v}=\frac{8 \pi G}{c^{4}} T_{\mu v(n, \psi \alpha \beta)}
$$

where the requirement of null rest mass

$$
m_{G}=0
$$

is implemented by the condition

$$
T_{\mu\left(n, \psi_{\alpha \beta}\right)}^{\mu}=0 .
$$

Thence, it follows that

$$
\begin{gathered}
R=-\frac{4 \pi G}{c^{4}} T_{\alpha \mu v_{\left(n, \psi_{\mu \nu}\right)}}^{\alpha}=0 \\
R_{\mu v}=\frac{8 \pi G}{c^{4}} T_{\left.\mu v_{(n, \psi \mu \nu}\right)} \\
\left(\partial^{\kappa} \psi_{\mu \nu}\right)_{; \kappa}=0 .
\end{gathered}
$$

The Lagrangean and the energy impulse tensor density for $\psi_{\alpha \beta}$, obeying to (19), respectively read

$$
L=-\hbar g_{\mu v} k^{v} \dot{q}^{\mu}=-\frac{\hbar c}{\omega} g_{\mu v} k^{v} k^{\mu}
$$

and

$$
T_{\mu \nu}=-\psi_{\alpha \beta} \psi^{* \alpha \beta} \frac{\hbar c^{2}}{\omega}\left(g_{v \alpha} k_{\mu} k^{\alpha}-g_{\alpha \beta} k^{\beta} k^{\alpha} g_{\mu \nu}\right)
$$

where

$$
\begin{gathered}
k_{\mu}=-\frac{1}{\hbar} \partial_{\mu} S \\
S=\frac{\hbar}{2 i} \ln \left[\frac{\psi_{\alpha \beta}}{\psi_{\alpha \beta}{ }^{*}}\right] .
\end{gathered}
$$

The energy-impulse tensor density of the field $\psi_{\alpha \beta}$ (21) in the low gravity limit [12] (by using (A1) in Appendix A) reads

$$
\begin{aligned}
T_{\mu v} & =-\psi_{\alpha \beta} \psi^{* \alpha \beta} \frac{\hbar c^{2}}{\omega}\left(g_{0 v \alpha} k_{\mu} k^{\alpha}+\varepsilon_{v \alpha} k_{\mu} k^{\alpha}-k_{\alpha} k^{\alpha} g_{0 \mu \nu}-k_{\alpha} k^{\alpha} \varepsilon_{\mu v}\right) \\
& =T_{0 \mu \nu}+|\psi|^{2} \frac{\hbar c^{2}}{\omega} k_{\alpha} k^{\alpha} \varepsilon_{\mu v}
\end{aligned}
$$

where $T_{0 \mu \nu}$ contains the zero and the first order terms of $\varepsilon_{\mu v}$, so that the residual second order of the Ricci tensor $R_{\mu \nu}$ (the term $k_{\alpha} k^{\alpha}$ is a first order term in $\varepsilon_{\mu \nu}$ ) reads

$$
R_{\mu v}+\frac{8 \pi G}{c^{4}} T_{0 \mu \nu}=-\frac{8 \pi G}{c^{4}} t_{\mu \nu}=-\frac{8 \pi G}{c^{4}}|\psi|^{2} \frac{\hbar c^{2}}{\omega} k_{\alpha} k^{\alpha} \varepsilon_{\mu v} \cong 0 .
$$

Moreover, by comparing (25) with the relation between the Ricci tensor and the metric tensor of weak gravitational waves [16] that reads 


$$
R_{\mu \nu}=-\frac{1}{2} g_{0}{ }^{\kappa \lambda} \frac{\partial^{2} \varepsilon_{\mu \nu}}{\partial q^{\kappa} \partial q^{\lambda}} \cong 0
$$

and by posing

$$
\psi_{\mu v}=A_{\mu \nu}^{\alpha \beta} \varepsilon_{\alpha \beta} \cong A \delta_{\mu}^{\alpha} \delta_{v}^{\beta} \varepsilon_{\alpha \beta}=A \varepsilon_{\mu v},
$$

for oscillating solutions of the form

$$
\varepsilon_{\mu \nu}=\varepsilon_{0 \mu \nu} \exp -i\left(k_{\alpha} q^{\alpha}+\varepsilon_{\alpha \beta} k^{\alpha} q^{\beta}\right) \cong \varepsilon_{0 \mu \nu} \exp -i k_{\alpha} q^{\alpha}
$$

it follows that

$$
R_{\mu \nu} \cong-\frac{1}{2} k_{\lambda} k^{\lambda} \varepsilon_{\mu \nu} \cong-\frac{8 \pi G}{c^{4}}|\psi|^{2} \frac{\hbar c^{2}}{\omega} k_{\alpha} k^{\alpha} \varepsilon_{\mu \nu}
$$

so that for the graviton field we obtain

$$
|\psi|^{2}=\frac{\omega}{\hbar} \frac{c^{2}}{16 \pi G}
$$

that by (24) leads to

$$
A=\frac{\sqrt{\omega}}{\left|\varepsilon_{0}\right|} \sqrt{\frac{c^{2}}{\hbar 16 \pi G}}
$$

and, at zero order, to

$$
\psi_{\mu v}=\sqrt{\omega} \sqrt{\frac{c^{2}}{\hbar 16 \pi G}} \frac{\varepsilon_{0 \mu v}}{\left|\varepsilon_{0}\right|} \exp -i k_{\alpha} q^{\alpha}=\frac{\sqrt{\omega c}}{\hbar} m_{p} \frac{\varepsilon_{0 \mu v}}{\left|\varepsilon_{0}\right|} \exp -i k_{\alpha} q^{\alpha}
$$

where $m_{p}=\sqrt{\frac{\hbar c}{16 \pi G}}$ is the Planck mass and where the EITD of the field

$$
t_{\mu \nu}=\frac{c^{4}}{16 \pi G} k_{\alpha} k^{\alpha} \varepsilon_{\mu v}
$$

correctly gives the energy-impulse pseudo-tensor of the plane waves of gravitational field (we do not need to distinguish between matter fields EITD and gravitational energy-impulse pseudo-tensor density given that also the gravity has its own field).

The dependence of the graviton field amplitude by $\sqrt{\omega}$ must not surprise ourselves given that the EITD increases larger is the frequency of its waves. Moreover, given that for the KGE it holds that $k_{\alpha} k^{\alpha}=\frac{\omega^{2}}{c^{2}}-k^{2}=\frac{m^{2} c^{2}}{\hbar^{2}}$ the rest mass of the graviton can be assumed null (at least at the first order (see (25)).

\section{Quantization of Gravitational Waves}

The quantization of the graviton field $\psi_{\mu \nu}$ and hence of the gravitational waves $\varepsilon_{\mu v}$, obeying to the equation

$$
\left(\partial^{\kappa} \varepsilon_{\mu \nu}\right)_{; \kappa}=\left(\partial^{K} \psi_{\mu \nu}\right)_{; \kappa}=0
$$

That, at the zero order, reads

$$
\partial_{\kappa} \partial^{\kappa} \psi_{\mu \nu}=0
$$


can be obtained by applying the covariant commutation rules [11] [12] (see Appendix B) that, in the limit of weak gravitational waves, are given by those of the zero order of the Minkowskian case.

$$
\begin{gathered}
{\left[\Psi_{\mu \kappa(q, t)}, \Psi_{\nu \lambda\left(q^{\prime}, t\right)}\right]=0} \\
{\left[\pi_{\mu \kappa(q, t)}, \pi_{\nu \lambda\left(q^{\prime}, t\right)}\right]=0} \\
{\left[\pi_{\mu \kappa(q, t)}, \Psi_{\left(q^{\prime}, t\right)}^{\nu \lambda}\right]=-i \hbar \delta^{(3)}\left(q-q^{\prime}\right) \delta_{\mu}^{\nu} \delta_{\kappa}^{\lambda}}
\end{gathered}
$$

where $\pi_{\mu v(q, t)}=\frac{\partial \mathcal{L}_{f 0}}{\partial \dot{\Psi}_{(q, t)}^{\mu v}}$, where $\mathcal{L}_{f 0}=-\frac{1}{2} \psi^{\dagger}{ }_{\alpha \beta} \partial_{\mu} \partial^{\mu} \psi^{\alpha \beta}$ is the zero order of the field Lagrangean

$$
\begin{aligned}
\mathcal{L}_{f} & =-\frac{1}{2} \psi_{\alpha \beta}^{\dagger}\left(\partial_{\mu} \partial^{\mu}+\partial_{\mu} \varepsilon^{\mu v} \partial_{\nu}\right) \psi^{\alpha \beta} \\
& =\frac{1}{2}\left(\partial_{\mu} \psi_{\alpha \beta}^{\dagger}\right)\left(\partial^{\mu} \psi^{\alpha \beta}\right)+\frac{1}{2}\left(\partial_{\mu} \psi_{\alpha \beta}^{\dagger}\right) \varepsilon^{\mu v}\left(\partial_{\nu} \psi^{\alpha \beta}\right) \\
& =\mathcal{L}_{f 0}-\frac{1}{2} \psi^{\dagger}{ }_{\alpha \beta} \partial_{\mu} \varepsilon^{\mu \nu} \partial_{\nu} \psi^{\alpha \beta}+\frac{1}{2}\left(\partial_{\mu} \psi_{\alpha \beta}^{\dagger}\right) \varepsilon^{\mu v}\left(\partial_{\nu} \psi^{\alpha \beta}\right)
\end{aligned}
$$

Thence, given the graviton field tensor

$$
\psi_{\mu \nu}=\int \frac{\mathrm{d}^{3} k}{(2 \pi)^{3}} \frac{1}{2 \omega_{k}}\left(a_{(k) \mu \nu} \mathrm{e}^{i k_{\beta} q^{\beta}}+a_{(k) \mu \nu}^{\dagger} \mathrm{e}^{-i k_{\beta} q^{\beta}}\right)
$$

it follows that the field Hamiltonian reads [16]

$$
H=\int \frac{\mathrm{d}^{3} k}{(2 \pi)^{3}} \frac{\hbar}{2}\left(a_{(k) \mu v} a_{(k)}^{\dagger \mu v}+a_{(k)}^{\dagger \mu v} a_{(k) \mu v}\right)
$$

that by the commutator identity

$$
\left[a_{(k) \mu v}, a_{\left(k^{\prime}\right)}^{\dagger}{ }^{\kappa \lambda}\right]=(2 \pi)^{3} 2 \hbar \omega_{k} \delta^{(3)}\left(k-k^{\prime}\right) \delta_{\mu}^{\kappa} \delta_{v}^{\lambda}
$$

can be recognized as the sum of harmonic oscillators of energy $\hbar \omega_{k}$.

Moreover, for the gravitational wave

$$
\begin{aligned}
\varepsilon_{\mu \nu} & =\int \frac{\mathrm{d}^{3} k}{(2 \pi)^{3}} \frac{1}{2 \omega_{k}}\left(A_{(k) \mu \nu} \mathrm{e}^{i k_{\beta} q^{\beta}}+A_{(k) \mu \nu}^{\dagger} \mathrm{e}^{-i k_{\beta} q^{\beta}}\right) \\
& =\sqrt{\frac{\hbar 16 \pi G}{c^{2}}} \int \frac{\mathrm{d}^{3} k}{(2 \pi)^{3}} \frac{1}{2 \omega_{k}} \frac{1}{\sqrt{\omega_{k}}}\left(a_{(k) \mu \nu} \mathrm{e}^{i k_{\beta} q^{\beta}}+a_{(k) \mu \nu}^{\dagger} \mathrm{e}^{-i k_{\beta} q^{\beta}}\right)
\end{aligned}
$$

the quantization rule reads

$$
\left[A_{(k) \mu v}, A_{\left(k^{\prime}\right)}^{\dagger}{ }^{\kappa \lambda}\right]=\frac{32 \pi G}{c^{2}}(2 \pi)^{3} \hbar^{2} \delta^{(3)}\left(k-k^{\prime}\right) \delta_{\mu}^{\kappa} \delta_{v}^{\lambda}
$$

that for the metric tensor wave leads to

$$
\begin{aligned}
& {\left[\dot{\varepsilon}_{\mu k(q, t)}, \varepsilon_{\left(q^{\prime}, t\right)}^{\nu \lambda}\right]} \\
& =\int_{0}^{\infty} \frac{\mathrm{d}^{3} k}{(2 \pi)^{3}} \frac{\mathrm{d}^{3} k^{\prime}}{(2 \pi)^{3}} \frac{1}{4 \omega_{k^{\prime}}}\left(A_{(k) \mu \nu} \mathrm{e}^{i k \cdot q}+A_{(k) \mu \nu}^{\dagger} \mathrm{e}^{-i k \cdot q}\right)\left(A_{\left(k^{\prime}\right)} \nu \lambda \mathrm{e}^{-i k^{\prime} \cdot q^{\prime}}+A_{\left(k^{\prime}\right)}^{\dagger \nu \lambda} \mathrm{e}^{-i k^{\prime} \cdot q^{\prime}}\right)
\end{aligned}
$$




$$
\begin{aligned}
& -\int_{0}^{\infty} \frac{\mathrm{d}^{3} k}{(2 \pi)^{3}} \frac{\mathrm{d}^{3} k^{\prime}}{(2 \pi)^{3}} \frac{1}{4 \omega_{k^{\prime}}}\left(A_{\left(k^{\prime}\right)}^{\nu \lambda} \mathrm{e}^{i k^{\prime} \cdot q^{\prime}}+A_{\left(k^{\prime}\right)}^{\dagger} \nu \lambda \mathrm{e}^{-i k^{\prime} \cdot q^{\prime}}\right)\left(A_{(k) \mu v} \mathrm{e}^{i k \cdot q}+A_{(k) \mu v}^{\dagger} \mathrm{e}^{-i k \cdot q}\right) \\
& =\int_{0}^{\infty} \frac{\mathrm{d}^{3} k}{(2 \pi)^{3}} \frac{\mathrm{d}^{3} k^{\prime}}{(2 \pi)^{3}} \frac{1}{4 \omega_{k^{\prime}}}\left(\left[A_{(k) \mu v}, A_{\left(k^{\prime}\right)}^{\dagger} \nu \lambda\right] \mathrm{e}^{i k \cdot q} \mathrm{e}^{-i k^{\prime} \cdot q^{\prime}}+\left[A_{(k) \mu v}^{\dagger}, A_{\left(k^{\prime}\right)}^{\nu \lambda}\right] \mathrm{e}^{-i k \cdot q} \mathrm{e}^{i k^{\prime} \cdot q^{\prime}}\right) \\
& =\frac{32 \pi G}{c^{2}}(2 \pi)^{3} \hbar^{2} \delta_{\mu}^{\kappa} \delta_{v}^{\lambda} \int_{0}^{\infty} \frac{\mathrm{d}^{3} k}{(2 \pi)^{3}} \frac{1}{\omega_{k}}\left(\mathrm{e}^{i k \cdot\left(q-q^{\prime}\right)}+\mathrm{e}^{-i k \cdot\left(q-q^{\prime}\right)}\right) \\
& =\frac{16 \pi G}{c^{2}}(2 \pi)^{3} \hbar^{2} \delta_{\mu}^{\kappa} \delta_{v}^{\lambda} \int_{-\infty}^{\infty} \frac{\mathrm{d}^{3} k}{(2 \pi)^{3}} \frac{1}{\omega_{k}} \mathrm{e}^{i k \cdot\left(q-q^{\prime}\right)} \\
& =\frac{16 \pi G}{c^{5}}(2 \pi) 3 \hbar^{2} \mathcal{F}^{-1}\left(\omega_{k}^{-1}\right)_{\left(q-q^{\prime}\right)} \delta_{\mu}^{\kappa} \delta_{v}^{\lambda}
\end{aligned}
$$

In the classical general relativity the gravitational waves can be whatever function of $k_{\mu} k^{\mu}$ [17] where the tensor $\varepsilon_{0 \mu v}$ for a wave propagating along $q_{1}$ has only four non-zero components: $\varepsilon_{023}, \varepsilon_{032}$ and $\varepsilon_{022}=-\varepsilon_{033}$ [17].

On the contrary, in the quantum case the QGE (18) is coupled to the quantum equation of the graviton field $\psi_{\mu \nu}$ (19), undergoing quantization, that is composed by quantized plane waves of frequency $\omega$ and energy $\hbar \omega$.

Generally speaking, the field determines the configurations of the connected geometry of the space-time, while quantization makes the field, as well as the geometry of the space-time, observables undergoing quantum dispersion.

Under this light, only the quantum fields undergo quantization while the geometry of the space-time acquires the quantum characters through the gravity equation.

\section{Conclusions}

By using the gravity equation that takes into account the non-local interaction of the quantum potential, the commutation rules for the quantization of the graviton field in the limit of weak gravity has been derived.

In the non-Minkowskian quantum approach proposed, where the coupling between the gravitational equation and the fields is explicitly defined, a massless boson field can be associated to the graviton. The paper shows that the commutation relations of the metric tensor of the gravitational waves can be analytically derived by those of the associated graviton field.

\section{Conflicts of Interest}

The author declares no conflicts of interest regarding the publication of this paper.

\section{References}

[1] Rovelli, C. (2004) Quantum Gravity. Cambridge University Press, Cambridge. https://doi.org/10.1017/CBO9780511755804

[2] Bousso, R. (2002)The Holographic Principle. Reviews of Modern Physics, 74, 825. https://doi.org/10.1103/RevModPhys.74.825 
[3] Ashtekar, A. (2011) Introduction to Loop Quantum Gravity. General Relativity and Quantum Cosmology, arXiv:1201.4598.

[4] Rovelli, C. and Vidotto, F. (2014) Covariant Loop Quantum Gravity. Cambridge University Press, Cambridge. https://doi.org/10.1017/CBO9781107706910

[5] Thiemann, T. (2007) Modern Canonical Quantum General Relativity. Cambridge University Press, Cambridge. https://doi.org/10.1017/CBO9780511755682

[6] Giddings, S.B. (2013) Is String Theory a Theory of Quantum Gravity? Foundations of Physics, 43, 115-139. https://doi.org/10.1007/s10701-011-9612-x

[7] Dewitt, B.S. (1967) Quantum Theory of Gravity. III. Applications of the Covariant Theory. Review Journals Archive, 162, 1239.

[8] Gupta, S.N. (1952) Quantization of Einstein's Gravitational Field: General Treatment. Proceedings of the Physical Society. Section A, A65, 608. https://doi.org/10.1088/0370-1298/65/8/304

[9] Gupta. S.N. and Radford, S.F. (1980) Quantum Field-Theoretical Electromagnetic and Gravitational Two-Particle Potentials. Physical Review D, 21, Article ID: 2213. https://doi.org/10.1103/PhysRevD.21.2213

[10] Faddeev, L.D. and Popov. V.N. (1967) Feynman Diagrams for the Yang-Mills Field. Physics Letters B, 25, 29-30. https://doi.org/10.1016/0370-2693(67)90067-6

[11] Chiarelli, P. (2017) The Einstein Equation for Quantum Mechanical Systems Derived from the Minimum Action Principle in the Hydrodynamic Representation. Part I: Classical Fields, arXiv:1711.06093.

[12] Chiarelli. P. (2018) The Hydrodynamic Representation of Quantum Equations in Curved Space-Time and the Related Einstein Equation: The Adequate Gravity for Quantum Fields?

https://www.researchgate.net/publication/318420781_The_hydrodynamic_representat ion_of_quantum_equations_in_curved_space-time_and_the_related_Einstein_equati on_The_adequate_gravity_for_quantum_fields

[13] Carroll, S.M., Press, W.H. and Turner, E.L. (1992) The Cosmological Constant. Annual Review of Astronomy and Astrophysics, 30, 499-542. https://doi.org/10.1146/annurev.aa.30.090192.002435

[14] Zel'dovich, Y.B. (1968) The Cosmological Constant and the Theory of elementary. Soviet Physics Uspekhi, 11, 381-393. https://doi.org/10.1070/PU1968v011n03ABEH003927

[15] Bialyniki-Birula, I., Cieplak, M. and Kaminski, J. (1992) Theory of Quanta. Oxford University Press, Oxford, 87-115.

[16] Le Bellac, M. (1991) Quantum and Statistical Field Theory. Oxford Science Publication, Oxford, 315-337.

[17] Landau, L.D. and Lifšits, E.M. (1976) Course of Theoretical Physics. Italian Edition, Vol. 2, Butterworth-Heinemann, Oxford, 335-361. 


\section{Appendix A. Non-Minkowskian Field Quantization in Weak Gravity}

For the purpose of this section (i.e., quasi-Minkowskian space-time with particles very far from the Plancknian mass density

$$
\left.\frac{m_{p}}{l_{p}^{3}}=\frac{c^{5}}{\hbar G^{2}}\right)
$$

we analyze the quantization of the non-Minkowskian KGE at the first order in the metric tensor. By using the metric tensor

$$
g_{v \mu}=g_{0_{v \mu}}+\varepsilon_{\mu \nu}=\left[\begin{array}{cccc}
1 & 0 & 0 & 0 \\
0 & -1 & 0 & 0 \\
0 & 0 & -1 & 0 \\
0 & 0 & 0 & -1
\end{array}\right]+\varepsilon_{\mu v}
$$

with

$$
\varepsilon_{\mu \nu} \varepsilon^{\mu \nu}=|\varepsilon|^{2} \ll 1, \frac{\partial_{\alpha} \varepsilon^{\mu \nu} \partial^{\alpha} \varepsilon_{\mu \nu}}{|\varepsilon|^{2}} \sim|\bar{k}|^{2} \ll l_{p}^{-2} \sim \frac{c^{3}}{\hbar G}
$$

where $\bar{k}$ is the modulus of the largest spectral component of the metric tensor, it follows that the KGE reads

$$
\partial_{\mu} \partial^{\mu} \psi+\frac{m^{2} c^{2}}{\hbar^{2}} \psi \cong-\partial_{\mu} \varepsilon^{\mu v} \partial_{\nu} \psi
$$

leading to the derivative weak gravity source

$$
j_{(t, q)}=-\partial_{\mu} \varepsilon_{(t, q)}^{\mu v} \partial_{\nu} \psi_{(t, q)}
$$

with the Christoffel symbol that reads

$$
\Gamma_{v \mu}^{\alpha}=\frac{1}{2} g_{0}^{\alpha \beta}\left(\partial_{\mu} \varepsilon_{\beta \nu}+\partial_{\nu} \varepsilon_{\beta \mu}-\partial_{\beta} \varepsilon_{v \mu}\right)
$$

where, at zero order, the field and its Hamiltonian read [15], respectively,

$$
\psi_{0}=\int \frac{\mathrm{d}^{3} k}{(2 \pi)^{3} 2 \omega_{k}}\left(a_{0}(k) \mathrm{e}^{-i k q}+a_{0}^{\dagger}(k) \mathrm{e}^{i k q}\right)
$$

and

$$
H_{0}=-\int \frac{\mathrm{d}^{3} k}{(2 \pi)^{3} 2 \omega_{k}}\left(a_{0}^{\dagger}(k) a_{0}(k)+a_{0}(k) a_{0}^{\dagger}(k)\right)
$$

where

$$
\omega_{k}= \pm c \sqrt{k_{x}^{2}+k_{y}^{2}+k_{z}^{2}+\frac{m^{2} c^{2}}{\hbar^{2}}}
$$

and where $a_{0}(k)$ and $a_{0}^{\dagger}(k)$ are the free particle creation and annihilation operators, respectively that obey to the commutation rules [15]

$$
\begin{aligned}
& {\left[a_{0}(k), a_{0}\left(k^{\prime}\right)\right]=\left[a_{0}^{\dagger}(k), a_{0}^{\dagger}\left(k^{\prime}\right)\right]=0,} \\
& {\left[a_{0}^{\dagger}(k), a_{0}^{\dagger}\left(k^{\prime}\right)\right]=\hbar(2 \pi)^{3} 2 \omega_{k} \delta^{(3)}\left(k-k^{\prime}\right) .}
\end{aligned}
$$


By using (A4), the perturbation Hamiltonian $H_{1}$ in interaction picture (at zero order in $\left.g_{\mu v}\right)$ reads [15]

$$
H_{1}=-\int \mathrm{d}^{3} q j \psi_{0}=-\int \frac{\mathrm{d}^{3} k}{(2 \pi)^{3} 2 \omega_{k}}\left(a_{0}(k) j^{*}(t, k) \mathrm{e}^{-i \omega_{k} t}+a_{0}^{\dagger}(k) j(t, k) \mathrm{e}^{i \omega_{k} t}\right)(
$$

where

$$
\begin{aligned}
j(t, k) & =\int \mathrm{d}^{3} q j(t, q) \mathrm{e}^{-i k q}=-\int \mathrm{d}^{3} q \mathrm{e}^{-i k q} \partial_{\mu} \varepsilon^{\mu \nu} \partial_{\nu} \psi \\
& \cong-\int \mathrm{d}^{3} q \mathrm{e}^{-i k q} \int \frac{\mathrm{d}^{3} k}{(2 \pi)^{3} 2 \omega_{k}}\left(a_{0}(k) \partial_{\mu} \varepsilon^{\mu \nu} \partial_{\nu} \mathrm{e}^{-i k q}+a_{0}^{+}(k) \partial_{\mu} \varepsilon^{\mu v} \partial_{\nu} \mathrm{e}^{i k q}\right)
\end{aligned}
$$

Since the geometrical background of gravity affects the commutation rules of the quantum fields, beyond the zero order of approximation (of $g_{\mu \nu}$ ) the Christoffel symbol enters in the definition of the equation of field evolution. For a scalar field $\psi$, the commutators(see Appendix B) read

$$
\begin{gathered}
{\left[\psi_{(q, t)}, \psi_{\left(q^{\prime}, t\right)}\right]=0} \\
{\left[\pi_{(q, t)}, \psi_{\left(q^{\prime}, t\right)}\right]=i \hbar \delta^{(3)}\left(q-q^{\prime}\right) \frac{\partial \psi_{\left(q^{\prime}, t\right)}}{\partial \psi_{(q, t)}}=i \hbar \delta^{(3)}\left(q-q^{\prime}\right)} \\
{\left[\pi_{(q, t)}, \pi_{\left(q^{\prime}, t\right)}\right]=-i \hbar \delta^{(3)}\left(q-q^{\prime}\right) \frac{\partial \pi_{\left(q^{\prime}, t\right)}}{\partial \psi_{(q, t)}}}
\end{gathered}
$$

that by using the field Lagrangean

$$
\begin{aligned}
\mathcal{L}_{f} & =-\frac{1}{2} \psi\left(\partial_{\mu} \partial^{\mu}+\frac{m^{2} c^{2}}{\hbar^{2}}+\partial_{\mu} \varepsilon^{\mu v} \partial_{\nu}\right) \psi \\
& =\frac{1}{2}\left(\partial_{\mu} \psi\right)\left(\partial^{\mu} \psi\right)-\frac{1}{2} \frac{m^{2} c^{2}}{\hbar^{2}} \psi^{2}+\frac{1}{2}\left(\partial_{\mu} \psi\right) \varepsilon^{\mu v}\left(\partial_{\nu} \psi\right)
\end{aligned}
$$

and the related momentum expression

$$
\pi_{(q, t)}=\frac{\partial \mathcal{L}_{f}}{\partial \dot{\psi}_{(q, t)}}=\dot{\psi}_{(q, t)}+\varepsilon^{0 v} \partial_{\nu} \psi=\pi_{0(q, t)}+\Delta \pi_{(q, t)}
$$

leads to

$$
\left[\pi_{(q, t)}, \pi_{\left(q^{\prime}, t\right)}\right]=-i \hbar \delta^{(3)}\left(q-q^{\prime}\right) \frac{\partial \pi_{\left(q^{\prime}, t\right)}}{\partial \psi_{(q, t)}}=-i \hbar \delta^{(3)}\left(q-q^{\prime}\right) \frac{\partial \varepsilon_{(\psi, q, t)}^{0 \nu}}{\partial \psi_{(q, t)}} \partial_{\nu} \psi_{(q, t)} .
$$

where $\varepsilon_{(\psi, q, t)}^{0 v}$ is defined by the GE.

As a final comment, it is worth noting to observe that in the first order treatment of the quantum problem, as above, the condition of small metric tensor gradients is taken respect to the quantum microscopic scale (but not the classical macroscopic scale) and it is free from the requirement of decoherence condition typical of the macroscopicity.

Furthermore, given that the quantum scale usually is of order or smaller than $10^{-9} \mathrm{~m}$, the approximation can describe quite large "macroscopic" gravitational fields. 


\section{Appendix B. Quantization Rules in Non-Minkowskian Space-Time}

The covariance principle, that has been used in the first part of the paper as a criterion for defining the hydrodynamic motion equation in the non-Minkowskian space of general relativity (see Section 3.3 of the part I), also fixes the commutation rules for quantization in non-Minkowskian space-time. In fact, in order to have the covariant form of the Klein-Gordon equation, the association $p_{\mu} \equiv p_{\mu}{ }^{o p}=i \hbar \partial_{\mu}$ for the momentum operator accordingly changes to

$$
p_{\mu}^{o p} B_{v}=i \hbar B_{v ; \mu}
$$

or, generally speaking, for a tensor $\underbrace{B_{k \ldots \ldots \ldots j}}_{N}$ of N-th order, to

$$
p_{\mu}^{o p} B_{k_{1} \cdots k_{i} \cdots k_{N}} \equiv i \hbar\left(\frac{\partial B_{k_{1} \cdots k_{i} \cdots k_{N}}}{\partial q^{\mu}}-\sum_{i=1}^{N} \Gamma_{k_{i} \mu}^{m_{i}} B_{k_{1} \cdots m_{i} \cdots k_{N}}\right) .
$$

while for the displacement operator it still holds

$$
q_{\mu}^{o p}=q_{\mu} .
$$

By using (B2), it follows that the commutation rules read

$$
\left[p_{\mu}, B_{v}\right]=-i \hbar\left(\frac{\partial B_{v}}{\partial q^{\mu}}-\Gamma_{v \mu}^{m} B_{m}\right)=i \hbar B_{v ; \mu}
$$

and hence

$$
\begin{gathered}
{\left[p_{\mu}, q_{v}\right]=-i \hbar\left(\frac{\partial q_{v}}{\partial q^{\mu}}-\Gamma_{v \mu}^{m} q_{m}\right)=-i \hbar\left(g_{v \mu}-q^{\alpha} \frac{\partial g_{\alpha v}}{\partial q^{\mu}}-\Gamma_{v \mu}^{m} q_{m}\right)=-i \hbar q_{v ; \mu}} \\
{\left[p_{\mu}, p_{v}\right]=-i \hbar\left(\frac{\partial p_{v}}{\partial q^{\mu}}-\Gamma_{v \mu}^{m} p_{m}\right)=-i \hbar p_{v ; \mu}}
\end{gathered}
$$

and by (B3)

$$
\left[q_{\mu}, q_{v}\right]=0
$$

Given that for the Minkowskian limit $\Gamma_{k \beta}^{\mu} \rightarrow 0$ and $\frac{\partial g_{\alpha V}}{\partial q^{\mu}} \rightarrow 0$, so that

$$
p_{v ; \mu}=\frac{\partial p_{v}}{\partial q^{\mu}}-\Gamma_{k \beta}^{\mu} p^{\beta} \rightarrow 0
$$

and

$$
q_{v ; \mu}=\left(g_{v \mu}-q^{\alpha} \frac{\partial g_{\alpha \nu}}{\partial q^{\mu}}-\Gamma_{\nu \mu}^{m} q_{m}\right) \rightarrow \eta_{\nu \mu}=\left|\begin{array}{cccc}
1 & 0 & 0 & 0 \\
0 & -1 & 0 & 0 \\
0 & 0 & -1 & 0 \\
0 & 0 & 0 & -1
\end{array}\right|,
$$

the standard quantization conditions are recovered for the Minkowskian problem.

Non-Minkowskian commutation relation for the boson KGE field.

By using (B5)-(B7), for a vector boson field $\Psi_{\mu}$ and its conjugated momen- 
tum $\pi_{\mu}$, the commutation rules read

$$
\begin{gathered}
{\left[\Psi_{\mu(q, t)}, \Psi_{v\left(q^{\prime}, t\right)}\right]=0} \\
{\left[\pi_{\mu(q, t)}, \pi_{v\left(q^{\prime}, t\right)}\right]=-i \hbar \delta^{(3)}\left(q-q^{\prime}\right)\left(\frac{\partial \pi_{v\left(q^{\prime}, t\right)}}{\partial \Psi_{(q, t)}^{\mu}}-\Gamma_{v \mu\left(q^{\prime}, t\right)}^{m} \pi_{m\left(q^{\prime}, t\right)}\right)} \\
{\left[\pi_{\mu(q, t)}, \Psi_{v\left(q^{\prime}, t\right)}\right]=-i \hbar \delta^{(3)}\left(q-q^{\prime}\right)\left(\frac{\partial \Psi_{v\left(q^{\prime}, t\right)}}{\partial \Psi_{(q, t)}^{\mu}}-\Gamma_{v \mu\left(q^{\prime}, t\right)}^{m} \Psi_{m\left(q^{\prime}, t\right)}\right)} \\
=-i \hbar \delta^{(3)}\left(q-q^{\prime}\right)\left(g_{v \mu}-\Psi_{(q, t)}^{\alpha} \frac{\partial g_{\alpha v\left(q^{\prime}, t\right)}}{\partial \Psi_{(q, t)}^{\mu}}-\Gamma_{v \mu\left(q^{\prime}, t\right)}^{m} \Psi_{m\left(q^{\prime}, t\right)}\right)
\end{gathered}
$$

where

$$
\pi_{\mu(q, t)}=\frac{\partial \mathcal{L}_{f}}{\partial \dot{\Psi}_{(q, t)}^{\mu}}
$$

where $\mathcal{L}_{f}$ is the field Lagrangean. 